\title{
Effects of Long-Term Hydrogen Intervention on Plasma Metabolites and Gut Microbiome of Rats in Health Status
}

\section{Fei Xie}

Beijing University of Technology

\section{Xue Jiang}

Beijing University of Technology

Yang Yi

Beijing University of Technology

Zi-Jia Liu

Beijing University of Technology

\section{Chen Ma}

Beijing University of Technology

Jin He

Beijing University of Technology

Zhi-ming Xun

Beijing University of Technology

\section{Meng Wang}

Beijing University of Technology

Meng-yu Liu

Beijing University of Technology

Yao Mawulikplimi Adzavon

Beijing University of Technology

\section{Peng-xiang Zhao}

Beijing University of Technology

Xue-mei Ma ( $\nabla$ xmma@bjtu.edu.cn )

Beijing University of Technology

\section{Research Article}

Keywords: hydrogen, intervention, plasma, metabolites, microbiome, rats, health status, preventive, therapeutic applications 
DOl: https://doi.org/10.21203/rs.3.rs-770433/v1

License: (c) (1) This work is licensed under a Creative Commons Attribution 4.0 International License. Read Full License 

metabolites and gut microbiome of rats in health status

3 Fei Xie ${ }^{1,2,{ }^{*}}$, Xue Jiang ${ }^{1,2^{*}}$, Yang $\mathrm{Yi}^{1,2^{*}}$, Zi-Jia $\mathrm{Liu}^{1,2}$, Chen $\mathrm{Ma}^{1,2}$, Jin $\mathrm{He}^{1,2}$, Zhi-ming

4 Xun ${ }^{1,2}$, Meng Wang ${ }^{1,2}$, Meng-yu Liu' ${ }^{1,2}$, Yao Mawulikplimi Adzavon ${ }^{1,2}$, Peng-xiang $5 \quad$ Zhao ${ }^{1,2}$, Xue-mei Ma ${ }^{1,2 \#}$

61 College of Life Science and Bio-engineering, Beijing University of Technology, 7 Beijing 100124, China;

82 Beijing Molecular Hydrogen Research Center, Beijing 100124, China;

$9 \quad$ * These authors contributed equally to this work.

10 Co-authers' email addresses: Fei Xie: xiefei990815@bjut.edu.cn; Xue Jiang:

11 804623081@qq.com; Yang Yi: yiyangxiufeng@emails.bjut.edu.cn.

12 \# Correspondence to: Xuemei Ma. College of Life Science and Bio-engineering, 13 Beijing University of Technology, No.100, Pingleyuan, Chaoyang District, Beijing 14 100124, China. Email: xmma@bjut.edu.cn. 
The potential for preventive and therapeutic applications of $\mathrm{H}_{2}$ have now been confirmed in various disease. However, the effects of $\mathrm{H}_{2}$ on health status have not been fully elucidated. Our previous study reported changes in the body weight and 13 serum biochemical parameters during the six-month hydrogen intervention. To obtain a more comprehensive understanding of the effects of long-term hydrogen consumption, the plasma metabolome and gut microbiota were investigated in this study. Compared with the control group, 14 and 10 differential metabolites (DMs) were identified in hydrogen-rich water ( $\mathrm{HRW}$ ) and hydrogen inhalation $(\mathrm{HI})$ group, respectively. Pathway enrichment analysis showed that HRW intake mainly affected starch and sucrose metabolism, and DMs in $\mathrm{HI}$ group were mainly enriched in arginine biosynthesis. 16S rRNA gene sequencing showed that HRW intake induced significant changes in the structure of gut microbiota, while no marked bacterial community differences was observed in $\mathrm{HI}$ group. HRW intake mainly induced significant increase in the abundance of Lactobacillus, Ruminococcus, Clostridium XI, and decrease in Bacteroides. HI mainly induced decreased abundances of Blautia and Paraprevotella. The results of this study provide basic data for further research on hydrogen medicine. Determination of the effects of hydrogen intervention on microbiota profiles could also shed light on identification of mechanism underlying the biological effects of molecular hydrogen.

\section{Introduction}

Hydrogen $\left(\mathrm{H}_{2}\right)$ is the smallest and lightest gas molecule, which has been historically considered as a biologically inert molecule. Early in 1975, Dole et al. firstly reported the possible anti-cancer effect of hyperbaric treatment of $97.5 \%$ hydrogen gas in a mouse model of skin tumor [1]. However, medical researchers did not pay considerable attention to $\mathrm{H}_{2}$ until Ohsawa et al. reported that inhalation of $1-4 \% \mathrm{H}_{2}$ gas significantly attenuates cerebral ischemia-reperfusion injury in rats by selectively neutralizing hydroxyl radicals and peroxynitrite [2]. The potential for preventive and therapeutic applications of $\mathrm{H}_{2}$ have now been confirmed in more than 170 different human and animal-disease models [3]. Several biological mechanisms have been proposed, but none of them can fully explain the multiple biological functions of $\mathrm{H}_{2}$ [4].

In mammals, the gut microbiome forms a complex ecosystem consisting of a vast number of interacting bacteria, archaea, bacteriophages, eukaryotic virus and fungi, most of which are commensal or mutualistic microorganisms [5]. In the past decade the gut microbiota has been proved to play a profound role in the training of host immunity, digesting food, regulating gut endocrine function and neurological signalling, modifying drug action and metabolism, eliminating toxins and producing numerous compounds that influence the host [6]. At present, the research on the relationship between hydrogen consumption and gut microbiome is relatively limited. Most studies showed that hydrogen-rich water (HRW) could improve intestinal structural integrity 
and upregulation of butyrate-producing bacteria with ameliorated clinical features of gut microbiota disturbances [7]. However, these studies have been primarily focused on the modulatory effect of HRW consumption on intestinal flora in pathological conditions, whether administration of HRW regulates gut microbiome in healthy animals remains largely unknown. In addition, as another commonly used method of hydrogen consumption, whether hydrogen inhalation could also affect gut microbiome need to be further investigated.

One of the aims of the present study was to explore the possible regulatory effects of long term of HRW intake and hydrogen inhalation on gut microbiome. Our previous study showed that long term of HRW intake or hydrogen inhalation can influence some serum biochemical parameters of normal rats, indicating the potential modulatory effect of hydrogen consumption on metabolism in health status [8]. To further investigate the effect of hydrogen consumption on metabolism, LC-MS based pseudotargeted metabolomics analysis was performed to determine the changes in the levels of plasma metabolites. The relationship between differentially expressed plasma metabolites and altered gut microbiota was also investigated.

\section{Results}

Effects of hydrogen intervention on plasma metabolites of rats. To determine the effects of hydrogen intervention on plasma metabolites, LC-MS-based pseudotargeted metabolomics analysis was performed on fasting plasma samples. Eighty-six plasma metabolites were identified consisting of amino acids and their derivatives, intermediates in glycolysis and the citric acid cycle, lipid metabolites, nucleotide metabolites, urea cycle metabolites, carbohydrates, co-factors/vitamines, and hormones. The OPLS-DA model from the plasma metabolic profile showed a good discrimination between HRW and the control group ( $R 2 X=0.262, R 2 Y=0.991$, $\mathrm{Q} 2=0.645$, Figure $1 \mathrm{~A}$ and $1 \mathrm{D}), \mathrm{HI}$ and the control group $(\mathrm{R} 2 \mathrm{X}=0.269, \mathrm{R} 2 \mathrm{Y}=0.968$, $\mathrm{Q} 2=0.359$, Figure $1 \mathrm{~B}$ and $1 \mathrm{E}), \mathrm{HRW}$ and $\mathrm{HI}(\mathrm{R} 2 \mathrm{X}=0.325, \mathrm{R} 2 \mathrm{Y}=0.976, \mathrm{Q} 2=0.684$, Figure $1 \mathrm{C}$ and $1 \mathrm{~F})$.
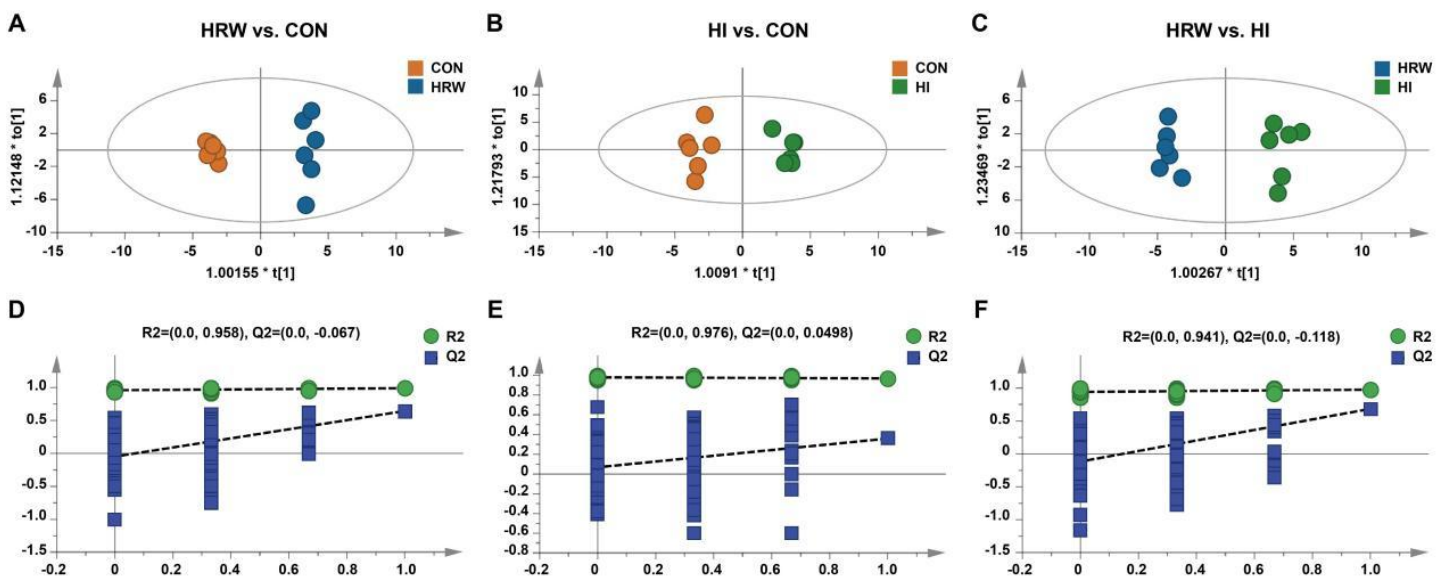

Figure 1 OPLS-DA models with corresponding values of R2X, R2Y, and Q2. (A) OPLS-DA 
score plot of HRW vs. CON; (B) OPLS-DA score plot of HI vs. CON; (C) OPLS-DA score plot of HRW vs. HI; (D-F) Validation plot obtained from 200 permutation tests, respectively.

Differential metabolites (DMs) were selected by using the cutoff of OPLS-DA VIP score $>1.0$ with a $P$ value $<0.05$ in the fold change of expression level between any two of the three groups. Thirty-five DMs were identified as shown in Supplementary Table 1. Compared with the control group, there are 14 and $10 \mathrm{DMs}$ in HRW and HI group respectively. Twenty-two DMs were identified between HRW and HI group. Compared the control group, all the DMs were down-regulated in HRW group, while all the DMs were down-regulated in $\mathrm{HI}$ group. Compared HRW group, all the DMs were up-regulated in $\mathrm{HI}$ group. As shown in Figure 2A, the dendrogram of hierarchical clustering showed the plasma samples in HRW group was clustered separately from the control group or $\mathrm{HI}$ group, however, the difference between $\mathrm{HI}$ group and the control group was much smaller. The pathway enrichment analysis based on metabolite quantitative alterations was performed by the MetaboAnalyst 5.0 (http://www.metaboanalyst.ca). The metabolic pathways with impact value $>0.1$ and $-\log (p)>2.0$ are considered the most relevant pathways involved in the conditions under study. The results showed that the DMs between HRW and control group were mainly concentrated in starch and sucrose metabolism (Figure 2B), the DMs between $\mathrm{HI}$ and control group were mainly involved in arginine biosynthesis (Figure 2C), the DMs between HRW and $\mathrm{HI}$ group were mainly enriched in glycerolipid metabolism, inositol phosphate metabolism, starch and sucrose metabolism, glyoxylate and dicarboxylate metabolism, and ascorbate and aldarate metabolism (Figure 2D). 


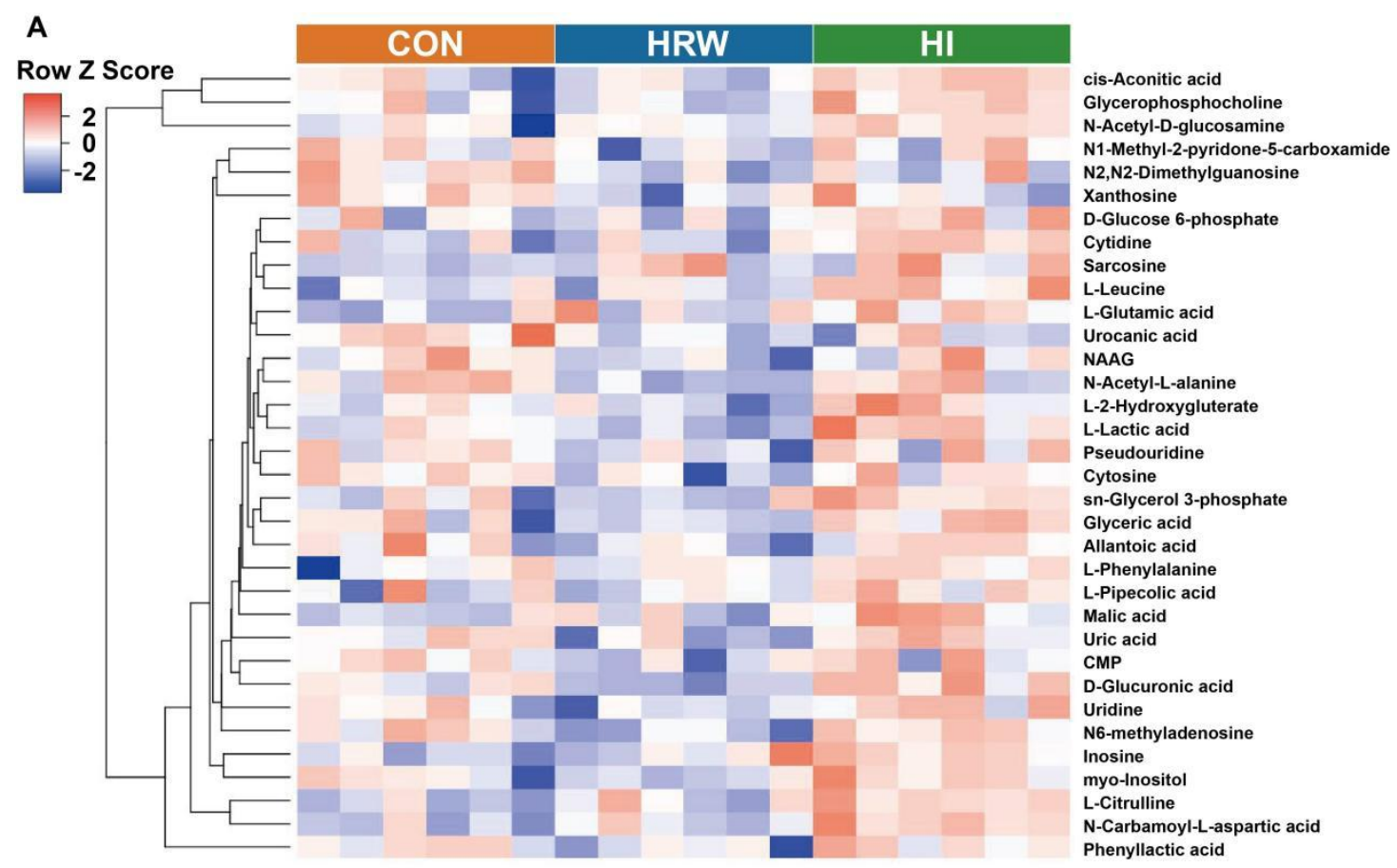

B

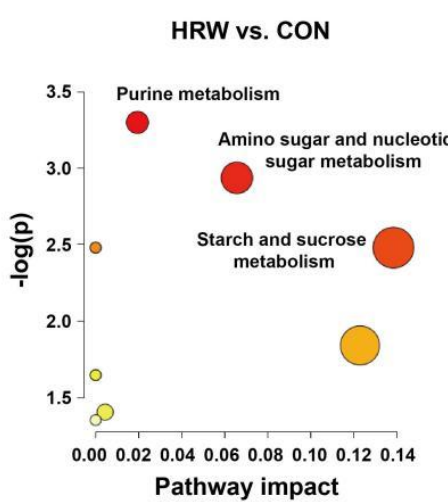

C

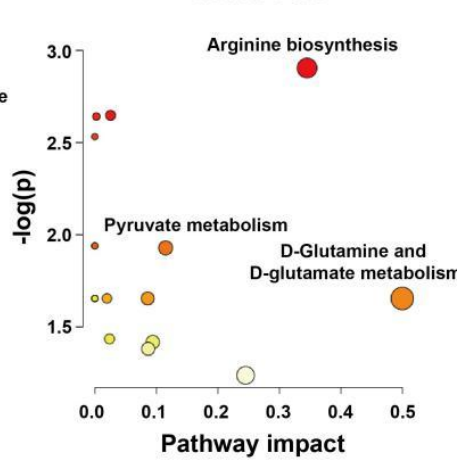

D

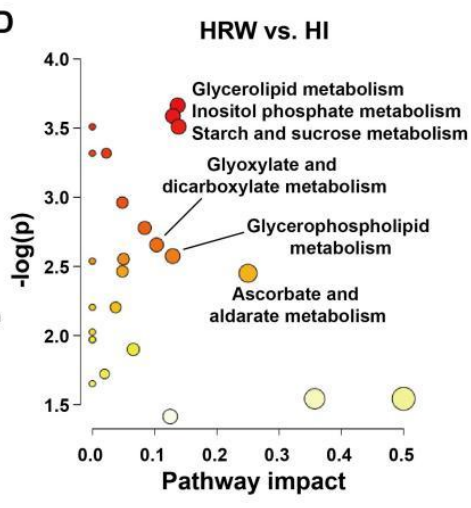

Figure 2 Analysis of differential metabolites in plasma samples among three groups. (A) The hierarchical clustering results for differential metabolites. (B-D) The functional enrichment analysis for differential metabolites between two groups. NAAG: $\mathrm{N}$-Acetylaspartylglutamic acid, CMP: Cytidine 5'-monophosphate.

Effects of hydrogen intervention on faecal microbiota profiles of rats. To investigate the effects of hydrogen intervention on faecal microbiota structure, we analyzed the bacterial communities on the samples by targeted 16S rRNA gene (V3-V4 region) sequencing using the Illumina MiSeq. Four measures of a-diversity (Chao1 (Figure 3A), PD whole tree (Figure 3B), Shannon (Figure 3C), and Simpson (Figure 3D)) all fail to report significant difference between the control group and HRW or $\mathrm{HI}$ group. However, the PD whole tree index was significantly higher in $\mathrm{HI}$ group than that in HRW group (Figure 3B). Analysis of similarities (ANOSIM) was performed to quantitatively compare the bacterial community differences between different groups. As shown in Figure 3E, ANOSIM revealed significant difference in the structure of gut microbiota between HRW and the control group (ANOSIM, $r=0.304$, 
$p=0.005)$. Significant bacterial community differences was also observed between HRW and $\mathrm{HI}$ group (Figure 3G, ANOSIM, r=0.369, $\mathrm{p}=0.003$ ). However, no significant difference was observed between $\mathrm{HI}$ and the control group (Figure 3F, ANOSIM, $r=-0.065, p=0.65$ ). The Wilcoxon rank-sum test was performed to identify differences in relative abundances of bacteria between the two groups. At the phyla levels, as shown in Figure $3 \mathrm{H}$, the dominant phyla of the three groups were Firmicutes, Bacteroidetes, Proteobacteria, and Actinobacteria. Compared with the controls, the relative percentage of Elusimicrobia was significantly increased in HRW group, while the proportion of Deferribacteres and Euryarchaeota were markedly decreased in HRW group. HI only induced markedly increase in the proportion of Elusimicrobia. Compared with HRW group, the proportion of Elusimicrobia was significantly lower and Spirochaetes and Euryarchaeota were markedly higher in $\mathrm{HI}$ group. At the family level, as shown in Figure 3l, the dominant family of the faecal microbiota were Prevotellaceae, Ruminococcaceae, Lachnospiraceae, and Lactobacillaceae (Figure 31). The abundances of Lactobacillaceae, Peptostreptococcaceae, and Elusimicrobiaceae were increased, and the abundances of Bacteroidaceae and Desulfovibrionaceae were decreased in HRW group compared with the controls. The decreased abundance in Porphyromonadaceae and increased abundance in Elusimicrobiaceae were observed in $\mathrm{HI}$ group compared with the controls. Compared with HRW group, the increased abundance in Acidaminococcaceae and decreased abundances in some other phyla (e.g., Lactobacillaceae, Porphyromonadaceae, Peptostreptococcaceae, and Sphingomonadaceae) were observed in $\mathrm{HI}$ group. At the genus levels, as shown in Supplementary Table 2, compared with the control group, eleven and seven genera exhibited significantly different abundances in HRW and $\mathrm{HI}$ group respectively. Compared with HRW group, twenty-three genera showed significant differences in relative abundances of bacteria in $\mathrm{HI}$ group. 
A

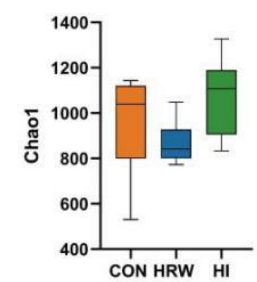

$E$

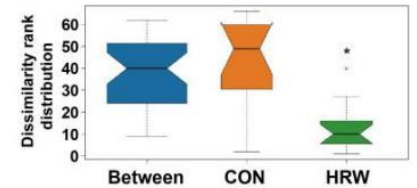

H

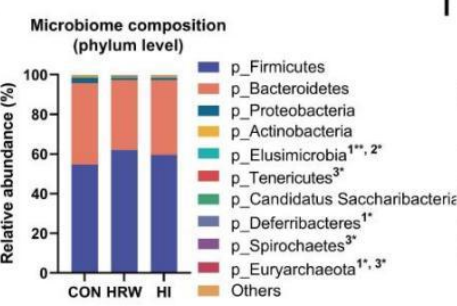

B

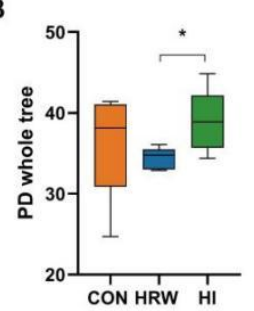

C

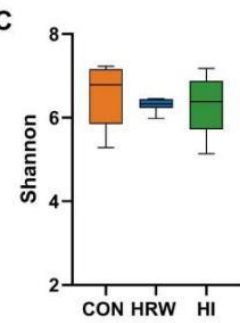

D

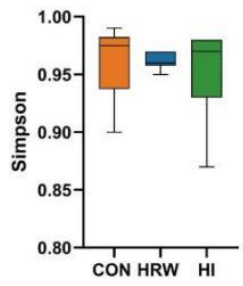

G

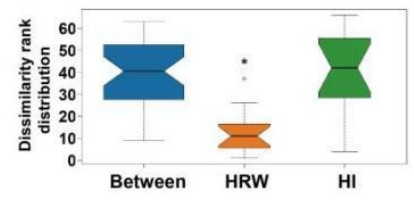

154 Figure 3 Analysis of bacterial community composition among three groups. (A-D) Alpha-diversity of bacterial community was measured by Chao1 (A), PD whole tree (B), Shannon (C), and Simpson (D); (E-G) ANOSIM was performed to quantitatively compare the bacterial community differences between different groups. (H-I) Community bar-plot analysis shows relative abundance of microbiota in each group at the phylum level $(H)$ and family level $(I), n=6$, in each group. 

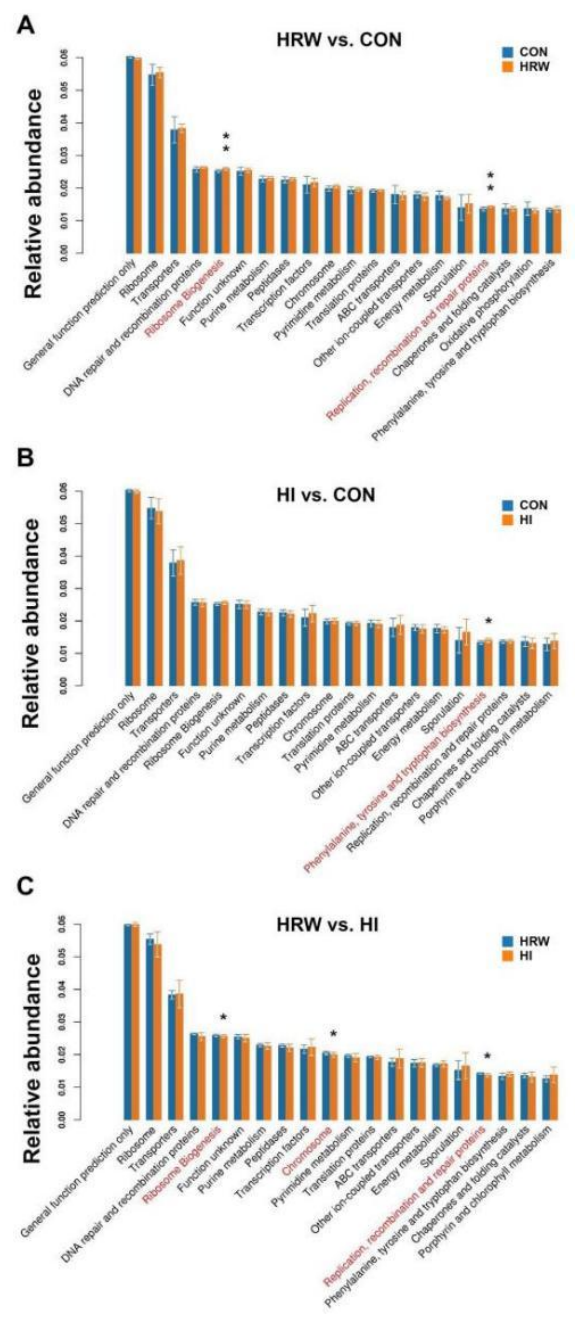

161 Figure 4. Differentially enriched KEGG pathways. The functional prediction at level-3 of KEGG pathway was performed by PICRUSt between HRW and Control group (A), $\mathrm{HI}$ and Control group (B), HRW and HI group (C).

To determine whether the changes in gut microbial taxa would alter the gut microbiota function, the functional prediction at level-3 of KEGG pathway was performed by PICRUSt. As shown in Figure 4, compared with the control group, the faecal microbiota of rats from HRW group had elevated pathways involved in ribosome biogenesis and replication, recombination and repair proteins. HI group had significantly enriched pathway involved in phenylalanine, tyrosine and tryptophan biosynthesis. Compared with HRW group, $\mathrm{HI}$ group had reduced pathways involved in ribosome biogenesis, chromosome, and recombination and repair proteins. 
A

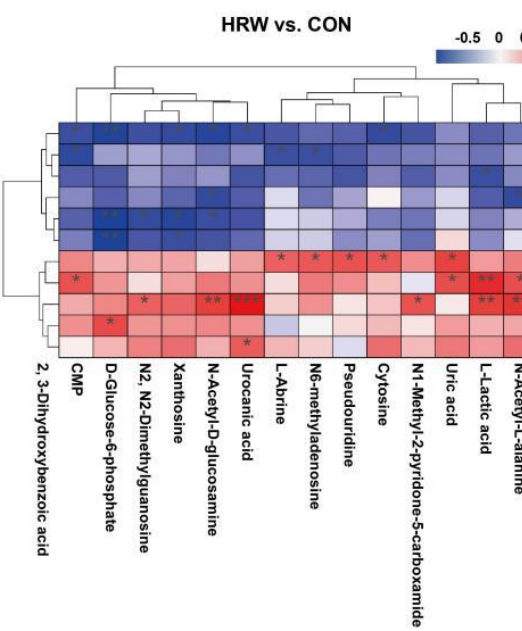

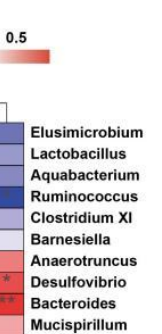
Mucispirillum
Bifidobacterium

B

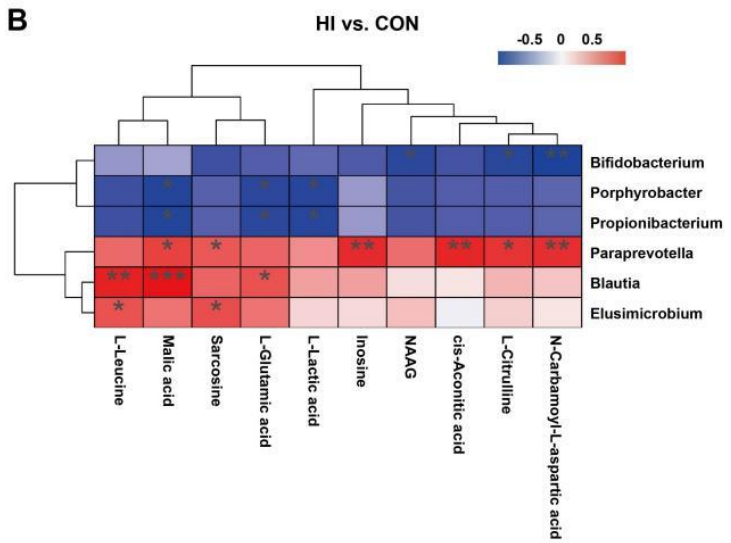

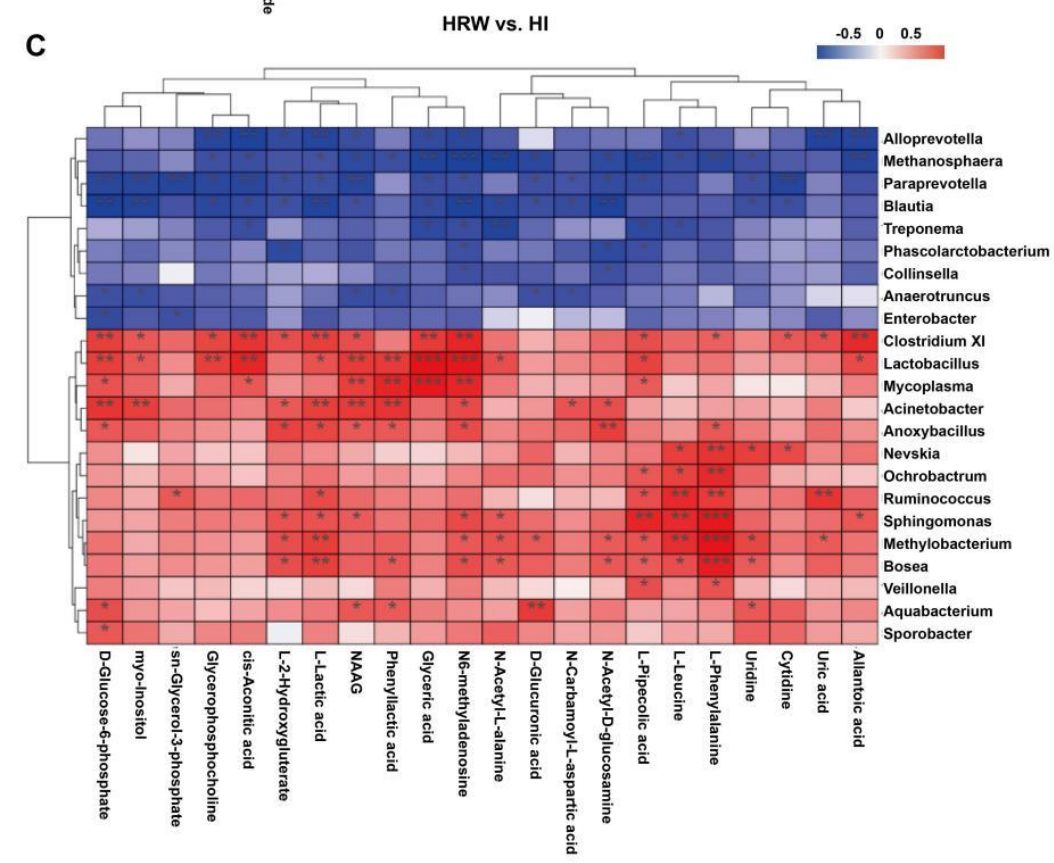

Figure 5. Correlation analyses between significantly different genera and differential plasma metabolites. Spearman's correlation test was performed between HRW and Control group (A), HI and Control group (B), HRW and HI group (C). 
Correlations between the faecal microbiota and plasma metabolites. To examine the possible relationship between differentially expressed plasma metabolites and altered gut microbiota at the genus level, multiomic analysis was performed by using Spearman's correlation test. The results of Spearman's correlation analysis between HRW and the control group are shown in Figure 5A, the abundance of urocanic acid had the strongest correlation with Bacteroides ( $r=0.944$, $\mathrm{p}<0.0001$ ). L-lactic acid showed comparable moderate positive correlations with Desulfovibrio and Bacteroides. N-Acetyl-L-alanine and N-Acetyl-D-glucosamine also showed moderate positive correlation with Bacteroides and Elusimicrobium. D-Glucose 6-phosphate showed moderate negative correlations with Elusimicrobium, Clostridium XI, and Barnesiella. Figure 5B showed the Spearman's correlation analysis between $\mathrm{HI}$ and the control group, malic acid had the strongest correlation with Blautia $(r=0.879, p=0.0002)$. L-leucine showed moderate positive correlation with Blautia. N-Carbamoyl-L-aspartic acid, cis-Aconitic acid, and inosine also showed moderate positive correlations with Paraprevotella. N-Carbamoyl-L-aspartic acid showed moderate negative correlation with Bifidobacterium. Figure $5 \mathrm{C}$ showed the Spearman's correlation analysis between HRW and $\mathrm{HI}$ group, L-phenylalanine showed high positive correlations with Sphingomonas $(r=0.866, p=0.0003)$, Methylocacterium $(r=0.921, p<0.0001)$, and Bosea $(r=0.848, p=0.0005)$. Glyceric acid also showed high positive correlations with Lactobacillus $(r=0.937, p<0.0001)$ and Mycoplasma ( $r=0.881, p=0.0002)$. N6-methyladenosine showed high positive correlation with Lactobacillus $(r=0.895, p<0.0001)$, while had strong negative correlation with Methanosphaera $(r=-0.834, p=0.0007)$.

\section{Discussion}

Our previous study reported for the first time that both HRW intake and $\mathrm{HI}$ can induce significant changes in several serum biochemical parameters in normal rats [8]. In this paper, to obtain a more comprehensive understanding of the metabolic alterations in response to HRW intake or HI, LC-MS based pseudotargeted metabolomics analysis was performed. The OPLS-DA models indicated clear separations between any two of the three groups based on their metabolomic responses. Compared with the control group, 14 and 10 DMs were identified in HRW and $\mathrm{HI}$ group, respectively. It is worth noting that all the DMs in HRW group were down-regulated, while all the DMs were up-regulated in $\mathrm{HI}$ group, $22 \mathrm{DMs}$ were identified between HRW and HI groups, indicating that the modulatory effects of HRW intake on metabolism differ markedly from $\mathrm{HI}$. Further functional enrichment analysis suggested the DMs were mainly involved in starch and sucrose metabolism and arginine biosynthesis in HRW and HI group, respectively.

Previous study showed that 4 weeks of HRW intake could siginificatly decrease the levels of blood glucose, lactate, and blood urea nitrogen (BUN) and exert antifatigue effects in chronic forced swimming mice [9]. It has also been reported that 3 months of HRW intake could markedly decrease the blood uric acid levels in male patients with hyperuricemia [10]. Consistent with these findings, our results also 
showed that 6 months of HRW intake could down-regulate plasma levels of D-glucose 6-phosphate, L-lactic acid, and uric acid. In addition, the plasma levels of nucleotides and their derivatives were also reduced after HRW intake, indicating the regulatory effects of HRW intake on nucleotides metabolism. Notably, all the changed nucleotides derivatives, inculding $\mathrm{m}^{6} \mathrm{~A}$, pseudouridine, and N2,N2-dimethylguanosine, were belong to RNA modifications. Among them, $\mathrm{m}^{6} \mathrm{~A}$ is the most widespread epigenetic modification on mammalian mRNA and has been shown to act as a key disease, including cancer, heart failure, viral infection, and type 2 diabetes [11, 12]. Pseudouridine was reported to undergo dynamic changes in response to serum starvation, hydrogen peroxide and heat shock in mammalian cells [11]. It has previously been reported that $\mathrm{H}_{2}$ administration could regulate expression of diverse genes [4], our results suggest that molecular hydrogen may regulate gene expression by affecting epigenetic modifications. In addition, the plasma levels of N1-methyl-2-pyridone-5-carboxamide was significantly decreased by HRW intake. Nicotinamide adenine dinucleotide $\left(\mathrm{NAD}^{+}\right)$is an important coenzyme for redox reactions, making it central to energy metabolism [13]. Nicotinamide mononucleotide (NMN) is one of the substrates for $\mathrm{NAD}^{+}$synthesis, which can be further metabolized to N1-methyl-2-pyridone-5-carboxamide. Thus, HRW intake may regulate energy metabolism via affecting $\mathrm{NAD}^{+}$synthesis.

Unlike HRW intake, HI had an up-regulatory effects on plasma DMs. Six of those DMs, including L-citrulline, L-leucine, sarcosine, L-glutamic acid, $\mathrm{N}$-carbamoyl-L-aspartic acid, and NAAG, were belong to amino acids and their derivatives. Among them, the plasma levels of L-Citrulline showed the most considerable increase in $\mathrm{HI}$ group compared to the controls. It has been showed in rats that only the intestine produced circulating citrulline, and the increased citrulline levels may be caused by either increased production or decreased utilization [14]. The decreased utilization could arise from a decrease in clearance, i.e. renal failure. However, no significant change of creatinine plasma levels was observed in our study, indicating that the increased citrulline levels was not caused by the impairment of renal function. Previous study showed that the increase in plasma citrulline was correlated with protein absorption improvement in patients with short bowel syndrome (SBS) followed in the first year after resection [15]. We supposed that the increased citrulline plasma levels may be assiociated with the improvement of enterocyte function, although this need to be further investigated. $\mathrm{HI}$ also induced a significant increase in NAAG plasma levels. NAAG is the most prevalent and widely distributed dipeptide in the mammalian nervous system [16]. The levels of NAAG in plasma and cerebrospinal fluid (CSF) were much lower than those in brain tissues [17]. It is well established that the increase in NAAG is neuroprotective against $\mathrm{N}$-methyl-D-aspartate (NMDA) receptor-mediated neurotoxicity, including ischemic brain injury [16]. HI was first reported to exert neuroprotective effects on ischemic stroke, and further studies also found its protective effects on other neurological impairment, including traumatic brain injury, subarachnoid hemorrhage, and neurodegenerative diseases [18]. Consistant with our rusults, the $\mathrm{HI}$ induced increase 
in NAAG was also observed in cortex tissues of mice with ischemic stroke [19]. Although the ability of molecular hydrogen to scavenge hydroxyl radicals may partly explain its neuroprotective effects, the regularory effects on NAAG may also responsible for its protective benefits. In addition, the significant increase in plasma levels of two citric acid cycle intermediates, cis-aconitic acid and malic acid, were also observed in our study, indicating that $\mathrm{HI}$ may accelerate mitochondrial energy metabolism. N-carbamoyl-L-aspartic acid and inosine are intermediates of pyrimidine and purine metabolism, respectively. The increase in the two metabolites suggest that $\mathrm{HI}$ may have modulatory effects on nucleoside metabolism. The plasma levels of other DMs, including L-leucine, sarcosine, L-glutamic acid, and L-lactic acid, varied slightly by $\mathrm{HI}$.

Recent studies have provided evidence that modulation of host gut microbiota may be one of the mechanisms contributing to the biological effects of exogenous hydrogen consumption. Qiu et al. showed that saturated hydrogen saline treatment could modulate the abundance of Bacteroides, Bifidobacteria, and Lactobacillus in feces, which may responsible for the improvement of lipid metabolism disorders in high-fat diet mice [20]. Jin et al. reported that sustained $\mathrm{H}_{2}$ release in the gut by hydrogen nanocapsule could increase the abundance of Akkermansia muciniphila and attenuate metabolic dysfunction-associated fatty liver disease [21]. In this study, $\mathrm{HRW}$ intake induced significant changes in the structure of gut microbiota, while no marked bacterial community differences was observed in HI group. Previous study showed that the peak of the hydrogen concentration in small intestine after oral intake of $5 \mathrm{ppm}$ of HRW was approximately 20 times higher than that after inhalation of $4 \%$ hydrogen gas [22]. The significant difference in hydrogen concentration in intestine between HRW intake and $\mathrm{HI}$ may contribute to the different effects on microbiota composition.

In our study, HRW intake induced significant increase in the proportion of Lactobacillus, Ruminococcus, Clostridium XI, Elusimicrobium, Barnesiella, and Aquabacterium, and decrease in Bacteroides, Anaerotruncus, Desulfovibrio, Mucispirillum, and Bifidobacterium. Lactobacillus and Bifidobacterium are the most common probiotic bacteria with the reported beneficial effects including aid digestion, reduce constipation, resist infections, prevent traveler's diarrhea and ameliorate intestinal bowel disease (IBD) [23]. In our study, HRW intake induced a significant increase in the abudance of Lactobacillus. Althrough HRW also induced a marked decrease in Bifidobacterium, the relative abundances of Bifidobacterium is very low. The increased abundance of Lactobacillus induced by HRW intake may contribute to the beneficial effects of HRW. It has been shown that supplementation with Ruminococcus flavefaciens could attenuate the antidepressant effects of duloxetine on depressive-like behavior [24], although the increased Ruminococcus has also been shown to be beneficial regarding antidepressant-induced constipation [24]. Previous study showed that 4 weeks of HRW intake could exert beneficial effects on depressive-like behavior in mice via suppression of the inflammasome activation [25], the antidepressive effects of HRW may be diminished by long-term HRW intake 
induced increase in Ruminococcus according to our study. Clostridium XI belongs to class Clostridia, which have been reported to attenuate inflammation and allergic diseases [26]. It has also been demonstrated that Clostridium species can utilize indigestible polysaccharide and produce lots of short-chain fatty acids (SCFAs), which are now considered as key players in the interactions with the host that impact on health and disease, especially given recent evidence for their capacity to modify the epigenome and effects on tissues and organs beyond the gut [27]. The increase in Clostridium XI derivied SCFAs may also contributed to the effects of HRW. In a study of 345 Chinese individuals, members of the genera Bacteroides has been shown to be more abundant in type II diabetic subjects compared to controls with normal glucose metabolism [28]. The improved glucose tolerance and hyperglycemia lowering effect of HRW intake have been previously reported [29, 30], which may be attributed by the HRW-induced decrease in Bacteroides levels. The results of the Spearman correlation analyses revealed a great number of significant correlations between the abundant of Bacteroides and DMs, including urocanic acid, L-lactic acid, $\mathrm{N}$-acetyl-D-glucosamine, and N-Acetyl-L-tyrosine, however, the causal relationships between alterations in Bacteroides abundance and plasma DMs need to be further investigated. Althrough the levels of other genera, including Elusimicrobium, Barnesiella, Aquabacterium, Anaerotruncus, Desulfovibrio, Mucispirillum, and Bifidobacterium changed significantly, their relative abundance was very low.

Compared with HRW group, the changes in fecal microbiota was found to be much less in $\mathrm{HI}$ group. Among these changed genera, the abundances of Blautia and Paraprevotella were significantly decreased. Althrough Blautia has been considered as a probiotic bacteria that occur widely in mammalian feces and intestines, a greater abundance of Blautia was found in irritable bowel syndrome and ulcerative colitis patients compared with healthy individuals [31]. The Paraprevotella genus is characterized by the production of succinic acid as major fermentation product, which can induce inflammation through the production of interleukin-1 $\beta$ [32]. Elevated succinic acid production has been reported in experimental models of hypertension and metabolic diseases [33]. The decreased abundances of Blautia and Paraprevotella may contributed to the effects of HI. Spearman correlation analyses revealed a great number of significant correlations between the abundant of Blautia and DMs, Correlation analyses revealed significant negative correlations between the abundance of Blautia and DMs, including L-leucine and malic acid. The abundance of Paraprevotella was negatively correlated with DMs, including inosine, cis-aconitic acid, and N-carbamoyl-L-aspartic acid. The other changed genera, including Elusimicrobium, Propionibacterium, Porphyrobacter, Methanosphaera, and Bifidobacterium, all had relatively low abundance.

One limitation of the present study is that research on the effects of hydrogen intervention on faecal microbiota profiles was focused on the genus level, and did not conduct in-depth studies at the species or even strain levels. Another limitation is the lack of proven causal relationships between alterations in microbiota profiles and plasma metabolites. In addition, further study should also evaluate the effects of 
hydrogen intervention on the production of SFCAs, which play a key role in microbiota-host interactions.

Collectively, the results of this study could provide basic data for further research on hydrogen medicine. Our results also shed light on the effects of different routes of hydrogen intervention on microbiota profiles, which may significantly contributed to the therapeutic effects of hydrogen in various diseases.

\section{Materials and Methods}

Animals and experimental design. Eighteen 3-week-old male Sprague-Dawley rats weighing 40-50 g were purchased from the Vital River Laboratory Animal Technology Co., Ltd (Beijing, China). Rats were housed under a constant temperature at $22{ }^{\circ} \mathrm{C}$ to $25{ }^{\circ} \mathrm{C}$ with a $12 \mathrm{~h}$ light-dark cycle and maintained on a normal diet. All procedures were approved by the Institutional Animal Experiment Committee of the Chinese PLA General Hospital and were conducted in compliance with the Regulations for the Administration of Affairs Concerning Experimental Animals (China) and the ARRIVE guidelines. Before the experiment, rats were adapted to laboratory conditions for one week. Rats were then randomly divided into three groups (6 in each group): (1) Control group: rats were maintained under normal conditions; (2) hydorgne-rich water (HRW) group: rats were given HRW by oral intake for $1 \mathrm{~h}$ each time, two times per day; (3) hydrogen inhalation $(\mathrm{HI})$ group: rats were treated with $\mathrm{HI}$ (4\%) for $1 \mathrm{~h}$ each time, two times per day. The experiment was last for six months. Fresh feces samples were collected via sterile operation before sacrifice, and were immediately stored at $-80{ }^{\circ} \mathrm{C}$ for microbiota analysis. After euthanasia, blood samples were immediately harvested from the portal vein. Plasma samples were obtained by centrifuging the whole blood at $1500 \mathrm{~g}$ for $10 \mathrm{~min}$ at $4{ }^{\circ} \mathrm{C}$, and stored at $-80{ }^{\circ} \mathrm{C}$ for pseudotargeted metabonomic analysis.

Hydrogen rich water preparation. $\mathrm{HRW}\left(\mathrm{H}_{2}\right.$ concentration $\left.>800 \mu \mathrm{M}\right)$ was kindly provided by Shenzhen Kelieng Biomedical Co. Ltd. (Shenzhen, China) and stored under atmospheric pressure at $23 \pm 2{ }^{\circ} \mathrm{C}$ in an aluminum pot. The hydrogen concentration was monitored by using a hydrogen electrode (Unisense A/S, Aarhus, Denmark), ensured that the hydrogen concentration of HRW for rats was maintained above $800 \mu \mathrm{M}$.

Inhalation of hydrogen gas. Rats were placed in a transparent closed box $(72 \times 53 \times 45 \mathrm{~cm}$, length $\times$ width $\times$ height $)$ connected to the hydrogen gas generator which composed of an Oxy-Hydrogen Machine (SG-3000; Gang'an Health Management [Beijing] Co., Ltd., Beijing, China) and a gas mixer, allowed to spontaneous respiration $\left(4 \% \mathrm{H}_{2}, 96 \%\right.$ air containing $\left.21 \% \mathrm{O}_{2}\right)$ for $1 \mathrm{~h}$ each time, and two times per day. The concentration of hydrogen and oxygen in the closed box was monitored by Thermal trace GC ultra-gas chromatography (Thermo Fisher, MA, USA).

LC-MS based pseudotargeted metabolomics analysis. The plasma samples were thawed at $4{ }^{\circ} \mathrm{C}$ and thoroughly vortexed. For each sample, $100 \mu \mathrm{L}$ was taken 
and transferred into a $1.5 \mathrm{~mL}$ Eppendorf tube. Then $400 \mu \mathrm{L}$ ice-cold methanol / acetonitrile $(1: 1, \mathrm{v} / \mathrm{v})$ was added. The samples were mixed by vigorous vortexed, sonicated in an ice-cold water bath sonicator for $20 \mathrm{~min}$, and kept at $-20{ }^{\circ} \mathrm{C}$ for $1 \mathrm{~h}$. Next, the samples were centrifuged at $14,000 \mathrm{~g}$ for $20 \mathrm{~min}$ at $4{ }^{\circ} \mathrm{C}$. The supernatant was transferred into a glass sampling vial and vacuum-dried at $4{ }^{\circ} \mathrm{C}$. Then $100 \mu \mathrm{L}$ of acetonitrile / water $(1: 1, \mathrm{v} / \mathrm{v})$ was added before centrifugation at 14,000 $\mathrm{g}$ for $20 \mathrm{~min}$ at $4{ }^{\circ} \mathrm{C}$. The resultant supernatants were used for metabolomics analysis. The quality control (QC) samples were prepared by mixing equal volumes of all plasma samples with all other steps as described above.

The chromatographic separation of plasma was carried out on a Waters I-class liquid chromatography system with ACQUITY UPLC BEH Amide column (1.7 $\mu \mathrm{m}$, $2.1 \times 100 \mathrm{~mm}$ column, Waters). The mobile phase system consists of $25 \mathrm{mM}$ ammonium acetate $+25 \mathrm{mM}$ ammonia $(\mathrm{pH}$ 9.75) (A) and acetonitrile (B). Column temperature was maintained at $40{ }^{\circ} \mathrm{C}$, and flow rate was $0.3 \mathrm{~mL} / \mathrm{min}$. Sample injection volume was $2 \mu \mathrm{L}$. The linear gradient for mobile phase B was as follows: $0-1$ $\min 95 \% \mathrm{~B} ; 1-14 \mathrm{~min}, 95 \%$ to $65 \% ; 14-16 \mathrm{~min}, 65 \%$ to $40 \% ; 16-18 \mathrm{~min}, 40 \% \mathrm{~B}$; $18-18.1 \mathrm{~min}, 40 \%$ to $95 \% ; 18.1-23 \mathrm{~min}, 95 \% \mathrm{~B}$. The QC samples were injected at regular intervals (every 6 samples) throughout the analytical run.

Mass data acquisition was performed using a 5500 QqQ MS (AB SCIEX). Detection was performed through ESI positive and negative modes. The MS parameters were set as follows: sheath gas temperature, $350{ }^{\circ} \mathrm{C}$; dry gas temperature, $350{ }^{\circ} \mathrm{C}$; sheath gas flow, $11 \mathrm{~L} / \mathrm{min}$; dry gas flow, $10 \mathrm{~L} / \mathrm{min}$; capillary voltage, $4000 \mathrm{~V}$ or $-3500 \mathrm{~V}$ in positive or negative modes, respectively; nozzle voltage, $500 \mathrm{~V}$; and nebulizer pressure, 30 psi. Multiple reaction monitoring (MRM) mode was used for detection. The dwell time of each ion pair was $3 \mathrm{~ms}$, and the total cycle time was $1.263 \mathrm{~s}$. The original MRM-based metabolomics data were analyzed using MRMAnalyzer ( $R$ ) as previously reported [34].

Unsupervised principal component analysis (PCA) was first performed to visualize the general trends for all the samples. Then, a supervised partial least-squares discriminant analysis (PLS-DA) model was used to classify the samples and find the relevant variables related to the sample grouping. The model was validated with 200 random permutations to assess the predictive variation of the model. Variable importance in projections (VIP) scores obtained from the PLS-DA model were used to assess the contribution of each variable to the established model. Metabolites that had VIP $>1$ and $p<0.05$ are identified as significantly changed metabolites. MetaboAnalyst 5.0 (https://www.metaboanalyst.ca/) was used for the functional enrichment analysis of the disturbed metabolites.

16S rRNA gene sequencing and microbiota analysis. Total genomic DNA was isolated from feces using the QIAamp Fast DNA Stool Mini Kit (QIAGEN, Germany) following the manufacturer's instructions. The quality and quantity of the extracted DNA were measured using a NanoDrop ND-2000 spectrophotometer (Thermo Fisher Scientific, USA). The V3-V4 region of the 16S rRNA gene were amplified with the 
forward primer 5'-ACTCCTACGGGAGGCAGCA-3', and the reverse primer 5'-GGACTACHVGGGTWTCTAAT-3'. The sample-specific barcodes were added to the ends of the primers. The PCR program was set as follows: $98{ }^{\circ} \mathrm{C} 10 \mathrm{~min}, 25$ cycles of $98{ }^{\circ} \mathrm{C} 15 \mathrm{~s}, 55^{\circ} \mathrm{C} 30 \mathrm{~s}, 72{ }^{\circ} \mathrm{C} 30 \mathrm{~s}$, and $72{ }^{\circ} \mathrm{C} 5 \mathrm{~min}$. The products of amplification were purified and then sequenced on Illumina MiSeq platform with MiSeq Reagent Kit v3.

The sequencing data were analyzed using QIME package (version 1.9.1). High-quality paried-end reads (quality score $\geq 20$ ) were assembled into tags by using FLASH (version 1.2.11). Tags with $>97 \%$ sequences identity were clustered into operational taxonomic units (OUTs) using USEARCH (version 10.0). A representative sequence of each OTU was selected and subjected to BLAST to assign taxonomic classification using SILVA database (version 132).

The alpha diversity indices were calculated by QIIME (version 1.9.1). A one-way analysis of similarity (ANOSIM) was performed to determine the differences in bacterial communities among groups. The differences in Alpha diversity indexes and phyla, family, and genera relative abundances between groups were calculated by use of the Independent-sample $t$-test (for the normally distributed data) or Wilcoxon rank-sum test (for the non-normally distributed data). A p value $<0.05$ was considered statistically significant. Phylogenetic Investigation of Communities by Reconstruction of Unobserved States (PICRUSt) was used to obtain relative Kyoto Encyclopedia of Genes and Genomes (KEGG) pathway abundance information.

Spearman multi-omic correlation analysis. Spearman correlation between the relative abundance of genera and the level of plasma metabolites was calculated in $R$ software (version 3.2.1) and visualized using ComplexHeatmap package in $\mathrm{R}$ software (version 3.2.1).

\section{References}

1. Dole, M., Wilson, F. \& Fife, W. Hyperbaric hydrogen therapy: a possible treatment for cancer. Science 190, 152-154, https://doi.org/10.1126/science.1166304 (1975).

2. Ohsawa, I., et al. Hydrogen acts as a therapeutic antioxidant by selectively reducing cytotoxic oxygen radicals. Nat Med 13, 688-694, https://doi.org/10.1038/nm1577(2007).

3. Ichihara, M., et al. Beneficial biological effects and the underlying mechanisms of molecular hydrogen - comprehensive review of 321 original articles. Med Gas Res 5, 12, https://doi.org/10.1186/s13618-015-0035-1 (2015).

4. Ge, L., Yang, M., Yang, N., Yin, X., and Song, W. Molecular hydrogen: a preventive and therapeutic medical gas for various diseases. Oncotarget 8, 102653-102673, https://doi.org/10.18632/oncotarget.21130 (2017).

5. Lynch, S. V. and Pedersen, O. The human intestinal microbiome in health and disease. N Engl J Med 375, 2369-2379, https://doi.org/10.1056/NEJMra1600266 (2016). 
6. Fan, Y. and Pedersen, O. Gut microbiota in human metabolic health and disease. Nat Rev Microbiol 19, 55-71, https://doi.org/10.1038/s41579-020-0433-9 (2021).

7. Ostojic, S. M. Hydrogen-rich water as a modulator of gut microbiota? J Funct Foods 78, 104360, https://doi.org/10.1016/j.jff.2021.104360 (2021).

8. Xun, Z., et al. Effects of long-term hydrogen intervention on the physiological function of rats. Sci Rep 10, 18509, https://doi.org/10.1038/s41598-020-75492-w (2020).

9. Ara, J., et al. Hydrogen water drinking exerts antifatigue effects in chronic forced swimming mice via antioxidative and anti-inflammatory activities. Biomed Res Int 2018, 2571269, https://doi.org/10.1155/2018/2571269 (2018).

10. Zheng, Y., Han, C., Chen, Z., Zeng, Q. A study on the effect of hydrogen on the hyperuricemia in males. Chin $J$ Clin Healthe 20, 118-121, https://doi.org/10.3969/J.issn.1672-6790.2017.02.002 (2017).

11. Wiener, D. and Schwartz, S. The epitranscriptome beyond $\mathrm{m}^{6} \mathrm{~A}$. Nat Rev Genet 22, 119-131, https://doi.org/10.1038/s41576-020-00295-8 (2021).

12. He, P. C. and He, C. $m^{6} A$ RNA methylation: from mechanisms to therapeutic potential. EMBO J 40, e105977, https://doi.org/10.15252/embj.2020105977 (2021).

13. Katsyuba, E., Romani, M., Hofer, D., Auwerx, J. NAD+ homeostasis in health and disease. Nat Metab. 2, 9-31, https://doi.org/10.1038/s42255-019-0161-5 (2020).

14. Crenn, P., Messing, B., Cynober, L. Citrulline as a biomarker of intestinal failure due to enterocyte mass reduction. Clin Nutr 27, 328-339, https://doi.org/10.1016/j.clnu.2008.02.005 (2008).

15. Gong, J., et al. Serum citrulline is a simple quantitative marker for small intestinal enterocytes mass and absorption function in short bowel patients. J Surg Res 127, 177-182, https://doi.org/10.1016/j.jss.2005.04.004 (2005).

16. Khacho, P., Wang, B., Bergeron, R. The good and bad sides of NAAG. Adv Pharmacol 76, 311-349, https://doi.org/10.1016/bs.apha.2016.01.003 (2016).

17. Kinoshita, K., Arai, K., Kawaura, K., Hiyoshi, T., Yamaguchi, J. Development, validation, and application of a surrogate analyte method for determining $\mathrm{N}$-acetyl-L-aspartyl-L-glutamic acid levels in rat brain, plasma, and cerebrospinal fluid. $J$ Chromatogr B Analyt Technol Biomed Life Sci 1003, 1-11, https://doi.org/10.1016/j.jchromb.2015.09.005 (2015).

18. Chen, W., Zhang, H., Qin, S. Neuroprotective effects of molecular hydrogen: a critical review. Neurosci Bull 37, 389-404. https://doi.org/10.1007/s12264-020-00597-1 (2021).

19. Chen, L., et al. UPLC-QTOF/MS-based metabolomics reveals the protective mechanism of hydrogen on mice with ischemic stroke. Neurochem Res 44, 1950-1963, https://doi.org/10.1007/s11064-019-02829-x (2019).

20. Qiu, X., et al. Saturated hydrogen improves lipid metabolism disorders and 

https://doi.org/10.1177/1535370219898407 (2020).

509 21. Jin, Z., et al. Nanocapsule-mediated sustained $\mathrm{H}_{2}$ release in the gut ameliorates metabolic dysfunction-associated fatty liver disease. Biomaterials 276, 121030, https://doi.org/10.1016/j.biomaterials.2021.121030 (2021).

22. Liu, C., et al. Estimation of the hydrogen concentration in rat tissue using an airtight tube following the administration of hydrogen via various routes. Sci Rep 4, 5485, https://doi.org/10.1038/srep05485 (2014).

23. Danneskiold-Samsøe, N. B., et al. Interplay between food and gut microbiota in health and disease.

Food

Res

Int

115 ,

23-31, https://doi.org/10.1016/j.foodres.2018.07.043 (2019).

518 24. Lukić, I., et al. Antidepressants affect gut microbiota and Ruminococcus flavefaciens is able to abolish their effects on depressive-like behavior. Trans/ Psychiatry 9, 133, https://doi.org/10.1038/s41398-019-0466-x (2019).

25. Zhang, Y., et al. Effects of hydrogen-rich water on depressive-like behavior in mice. Sci Rep 6, 23742, https://doi.org/10.1038/srep23742 (2016).

26. Guo, P., Zhang, K., Ma, X., He, P. Clostridium species as probiotics: potentials and $\begin{array}{llllll}\text { challenges. } & J & \text { Anim } & \text { Sci }\end{array}$ https://doi.org/10.1186/s40104-019-0402-1 (2020).

27. van der Hee, B., Wells, J. M. Microbial regulation of host physiology by short-chain fatty acids. Trends Microbiol 29, 700-712, https://doi.org/10.1016/j.tim.2021.02.001 528 (2021).

28. Qin, J., et al. A metagenome-wide association study of gut microbiota in type 2 diabetes. Nature 490, 55-60, https://doi.org/10.1038/nature11450 (2012).

29. Kajiyama, S., et al. Supplementation of hydrogen-rich water improves lipid and glucose metabolism in patients with type 2 diabetes or impaired glucose tolerance. Nutr Res 28, 137-143, https://doi.org/10.1016/j.nutres.2008.01.008 (2008).

30. Kamimura, N., Nishimaki, K., Ohsawa, I., Ohta, S. Molecular hydrogen improves obesity and diabetes by inducing hepatic FGF21 and stimulating energy metabolism in db/db mice. Obesity 19, 1396-1403, https://doi.org/10.1038/oby.2011.6 (2011).

31. Liu, X., et al. Blautia-a new functional genus with potential probiotic properties? Gut Microbes 13, 1-21, https://doi.org/10.1080/19490976.2021.1875796 (2021). HIF-1a. Nature 496, 238-242, https://doi.org/10.1038/nature11986 (2013).

33. Sadagopan, N., et al. Circulating succinate is elevated in rodent models of 
544 34. Cai, Y., et al. An integrated targeted metabolomic platform for high-throughput

545 metabolite profiling and automated data processing. Metabolomics 11, 1575-1586,

546 https://doi.org/10.1007/s11306-015-0809-4 (2015).

548 Acknowledgements

549 This work was supported by Military Logistics Key Open Research Projects (BHJ17L018).

\section{Author contributions}

551 X.-m.M. and F.X. designed the overall project. F.X., X.J., Y.Y., Z.-j.L., and C.M. performed

552 the animal experiments. J.H. and Z.-m.X. performed the LC-MS based pseudotargeted 553 metabolomics analysis, M.W. and M.-y.L. performed the 16S rRNA gene sequencing, F.X. 554 analyzed the data and wrote the manuscript, Y.-m.A., P.-x.Z., and X.-m.M. revised the 555 manuscript.

556 Competing interests

557 The authors report no conflicts of interest in this work.

558 Availability of data and materials

559 The data that support the findings of this study are available from the corresponding 560 author upon reasonable request. 



\section{Supplementary Files}

This is a list of supplementary files associated with this preprint. Click to download.

- supplementarytables.pdf 